\title{
ACCP GUIDELINE
}

\section{Standards of Practice for Clinical Pharmacists}

\author{
American College of Clinical Pharmacy*
}

KeY Words clinical pharmacy, clinical pharmacist, standards of practice, direct patient care, board certification, pharmacy practice, professionalism, research, scholarship, collaborative drug therapy management.

(Pharmacotherapy 2014;34(8):794-797) doi: 10.1002/phar.1438

This document sets forth ACCP's expectations for clinical pharmacists within the United States and countries around the world where clinical pharmacy is emerging. It is also intended to serve as a reference for those designing and assessing clinical pharmacy education and training programs. In addition to articulating the clinical pharmacist's process of care and documentation, the eight standards listed here address the clinical pharmacist's involvement in collaborative, team-based practice and privileging; professional development and maintenance of competence; professionalism and ethics; research and scholarship; and other professional responsibilities. The standards define for the public, health professionals, and policy-makers what they can and should expect of clinical pharmacists.

\section{Qualifications}

Clinical pharmacists are practitioners who provide comprehensive medication management ${ }^{1}$ and related care for patients in all health care settings. They are licensed pharmacists with specialized advanced education and training who possess the clinical competencies necessary to practice in team-based, direct patient care

\footnotetext{
Approved by the American College of Clinical Pharmacy Board of Regents on January 22, 2014.

*Address for correspondence: American College of Clinical Pharmacy, 13000 W. 87th St. Parkway, Suite 100, Lenexa, KS 66215; e-mail: accp@accp.com.

(C) 2014 Pharmacotherapy Publications, Inc.
}

environments. ${ }^{2,3}$ Accredited residency training or equivalent postlicensure experience is required for entry into direct patient care practice. Board certification is also required once the clinical pharmacist meets the eligibility criteria specified by the Board of Pharmacy Specialties (BPS). ${ }^{4}$

\section{Process of Care}

Clinical pharmacists work in collaboration ${ }^{\mathrm{a}}$ with other providers to deliver comprehensive medication management that optimizes patient outcomes. Care is coordinated among providers and across systems of care as patients transition in and out of various settings.

The clinical pharmacist's process of care comprises the following components.

\section{Assessment of the Patient}

The clinical pharmacist assesses medicationrelated $^{\mathrm{b}}$ needs by:

\footnotetext{
a"Collaboration" in the context of this document refers to collaborative and cooperative practice activities performed by the clinical pharmacist as authorized by (i) state practice acts and (ii) formal collaborative drug therapy management agreements with other providers and/or conferred by local privileging within the relevant practice, health system, organization, or institution.

b"Medication-related" applies to issues pertaining to (i) the indication/absence of indication, use and administration, therapeutic goals, adverse drug events, drug interactions, and monitoring of medications; (ii) the patient's adherence, attitudes, beliefs, and preferences regarding his/her medications; and (iii) any allergies or adverse reactions to medications.
} 
- Reviewing the medical record using a problem-oriented framework (e.g., interpreting and analyzing subjective and objective information) to determine the clinical status of the patient;

- Meeting with the patient/caregivers to obtain and document a complete medication history to identify all of the patient's current medications $^{c}$ (including regimens and administration routes), medication-taking behaviors, adherence, allergies, and attitudes and experiences with medication therapy ${ }^{\mathrm{d}}$;

- Obtaining, organizing, and interpreting patient data; and

- Prioritizing patient problems and medication-related needs.

\section{Evaluation of Medication Therapy}

The clinical pharmacist identifies strategies to optimize medication therapy by:

- Assessing, with other members of the health care team, the appropriateness of current medications on the basis of health conditions, indication, and the therapeutic goals of each medication;

- Evaluating the effectiveness, safety, and affordability of each medication;

- Assessing medication-taking behaviors and adherence to each medication; and

- Identifying medication-related problems and evaluating collaboratively with other members of the health care team the need for intervention.

Development and Implementation of a Plan of Care

The clinical pharmacist develops and implements, collaboratively with the patient and his/ her health care providers, a plan for optimizing medication therapy by:

- Reviewing the patient's active medical problem list to inform and guide the development of an individualized assessment and plan for optimizing medication therapy;

\footnotetext{
"Medications" are defined as any of the following: prescription drugs, nonprescription drugs, vaccines, or complementary and alternative medications.

${ }^{\mathrm{d}}$ Some patient conditions and clinical settings (e.g., in an intensive care unit, mental health facility, or emergency department) may render this activity unfeasible.
}

- Formulating a comprehensive medication management assessment and plan ${ }^{\mathrm{e}}$ in collaboration with the health care team and implementing this plan to achieve patient-specific outcomes;

- Educating the patient/caregivers (both verbally and in writing) to ensure understanding of the care plan, to optimize adherence, and to improve therapeutic outcomes; and

- Establishing patient-specific measurable parameters and time frames for monitoring ${ }^{\mathrm{p}}$ and follow-up in collaboration with other members of the health care team.

\section{Follow-up Evaluation and Medication}

\section{Monitoring}

The clinical pharmacist performs follow-up evaluations in collaboration with other members of the health care team to continually assess patient outcomes by:

- Coordinating with other providers to ensure that patient follow-up and future encounters are aligned with the patient's medical and medication-related needs;

- Revisiting the medical record to obtain updates on the clinical status of the patient and then meeting with the patient/caregivers to obtain an updated medication history to identify, assess, and document any new medication-related needs or problems;

- Conducting ongoing assessments and refining the plan of care to optimize medication therapy and ensure that individual goals are achieved; and

- Monitoring, modifying, documenting, and managing the plan of care in collaboration with the patient/caregivers and his/her other health care providers.

\section{Documentation}

Clinical pharmacists document directly in the patient's medical record the medication-related

\footnotetext{
${ }^{\mathrm{e}} \mathrm{A}$ comprehensive medication management plan relies on coordinated, team-based collaboration to initiate, modify, monitor, and/or discontinue medication therapy. Implementation of components of this plan may be delegated to the clinical pharmacist through a collaborative drug therapy management agreement or other formalized management protocol.

${ }^{\mathrm{f}}$ In many settings, the clinical pharmacist will use tools that facilitate his/her monitoring of the patient (e.g., monitoring forms, flow sheets, and other aids to closely track and organize patient-specific data and/or data sets).
} 
assessment and plan of care to optimize patient outcomes. This documentation should be compliant with the accepted standards for documentation (and billing, where applicable) within the health system, health care facility, outpatient practice, or pharmacy in which one works. Where applicable, accepted standards must be considered as they relate to the use of electronic health records (EHRs), health information technology and exchange systems, and e-prescribing.

The following components of the encounter are essential to include in the documentation, which may be communicated in the form of a traditional SOAP (subjective data, objective data, assessment, plan) note or other framework consistent with the standards of documentation within the practice setting.

\section{Medication History}

- A brief summary of the patient's past medication use and related health problems as an introduction to the documentation that will follow;

- A listing of all current medications that includes information regarding actual use, adherence, and attitudes toward therapy; and

- A listing of medication-related allergies and any adverse drug events that may affect prescribing and monitoring or preclude the future use of a medication.

Active Problem List with an Assessment of Each Problem

- A listing of current health conditions and supporting data for the status of each condition, emphasizing associated medications and medication-related problems that may have an impact on desired goals; and

- A listing of any additional medication-related problems or other medical issues that may be unrelated to current health conditions.

Plan of Care to Optimize Medication Therapy and Improve Patient Outcomes

- The specific medication therapy plan that has been or will be implemented collaboratively by the health care team, including drug, dose, route, frequency, and relevant monitoring parameters; and
- The collaborative plan for follow-up evaluation and monitoring as well as future visits.

\section{Collaborative, Team-Based Practice and Privileging}

Clinical pharmacists work with other health professionals as members of the health care team to provide high-quality, coordinated, patientcentered care. ${ }^{5}$ They establish written collaborative drug therapy management agreements (CDTM) with individual physicians, medical groups, or health systems and/or hold formally granted clinical privileges from the medical staff or credentialing system of the organization in which they practice. ${ }^{4}$ These privileging processes, together with the applicable state pharmacy practice act, confer certain authorities, responsibilities, and accountabilities to the clinical pharmacist as a member of the health care team and contribute to the enhanced efficiency and effectiveness of team-based care.

\section{Professional Development and Maintenance of Competence}

Clinical pharmacists maintain competence in clinical problem-solving, judgment, and decision-making; communication and education; medical information evaluation and management; management of patient populations; and a broad range of therapeutic knowledge domains. ${ }^{6}$ Clinical pharmacists maintain competency through:

A. Certification and maintenance of certification in the appropriate specialty relevant to their practice, including those specialties recognized by the BPS or other nationally recognized multiprofessional certifications;

B. Consistent participation in continuing professional development (CPD) activities that enhance direct patient care practice abilities; and

C. Maintenance of active licensure, including required continuing pharmacy education activities, through the appropriate state board(s) of pharmacy.

Clinical pharmacists also pursue professional and career development by participating in formal and informal activities that enhance research and scholarship, teaching, leadership, and/or management. 


\section{Professionalism and Ethics}

Clinical pharmacists have a covenantal, "fiducial" relationship with their patients. This relationship relies on the trust placed in the clinical pharmacist by the patient and the commitment of the clinical pharmacist to act in the best interest of individual patients and patient populations, within the context of legal and ethical parameters. Clinical pharmacists exhibit the traits of professionalism: responsibility, commitment to excellence, respect for others, honesty and integrity, and care and compassion. ${ }^{7}$ They subscribe to the pharmacy profession's code of ethics and adhere to all pharmacist-related legal and ethical standards.

\section{Research and Scholarship}

Clinical pharmacists support and participate in research and scholarship to advance human health and health care by developing research questions; conducting or participating in clinical, translational, and health services research; contributing to the evolving literature in evidence-based pharmacotherapy; and/or disseminating and applying research findings that influence the quality of patient care.

\section{Other Responsibilities}

Clinical pharmacists serve as direct patient care providers, but they may also serve as educators, researchers, clinical preceptors/mentors, administrators, managers, policy developers, and consultants. As the clinical pharmacy discipline grows, it must continue to familiarize more patients, families, caregivers, other health professionals, payers/insurers, health care administrators, students, and trainees with the full range of clinical pharmacists' responsibilities.

\section{References}

1. Patient-Centered Primary Care Collaborative. The patient-centered medical home: integrating comprehensive medication management to optimize patient outcomes resource guide, 2nd ed. Washington, DC: Patient-Centered Primary Care Collaborative; 2012. Available from http://www.pcpcc.org/sites/default/ files/media/medmanagement.pdf. Accessed March 24, 2014.

2. Mitchell P, Wynia M, Golden R, et al. Core principles \& values of effective team-based health care. Washington, DC: Institute of Medicine; 2012. Available at www.nationalahec.org/pdfs/ VSRT-Team-Based-Care-Principles-Values.pdf. Accessed March 24, 2014.

3. American College of Clinical Pharmacy. The definition of clinical pharmacy. Pharmacotherapy 2008;28:817-8. Available from http://www.accp.com/docs/positions/commentaries/Clinpharm defnfinal.pdf. Accessed March 24, 2014.

4. American College of Clinical Pharmacy. Qualifications of pharmacists who provide direct patient care: perspectives on the need for residency training and board certification. Pharmacotherapy 2013;33:888-91. Available from http://www.accp.com/ docs/positions/commentaries/Commntry_BOR_DPC_phar1285.pdf. Accessed March 24, 2014.

5. Doherty RB, Crowley RA. Principles supporting dynamic clinical care teams: an American College of Physicians position paper. Ann Intern Med. doi:10.7326/0003-4819-159-9-201311050-00710. Available from http://annals.org/article.aspx?articleid=1737233. Accessed March 24, 2014.

6. Burke JM, Miller WA, Spencer AP, et al. Clinical pharmacist competencies. Pharmacotherapy 2008;28:806-15. Available from http://www.accp.com/docs/positions/whitePapers/Clini PharmCompTFfinalDraft.pdf. Accessed March 24, 2014.

7. Roth MT, Zlatic TD. Development of student professionalism. Pharmacotherapy 2009;29:749-56. Available from http:// www.accp.com/docs/positions/whitePapers/devstuprof.pdf. Accessed March 24, 2014. 